\title{
Assessment of quadricuspid aortic valve with real time three-dimensional transthoracic echocardiography
}

Janez Poklukar*, Mitja Lainscak², David Zizek ${ }^{3}$, Jernej Markes', Nadja Ruzic Medvescek³, Robert Marcun', Marko Sluga', Pavel Berden ${ }^{3}$

'Jesenice General Hospital, Jesenice, Slovenia

${ }^{2}$ University Clinic Golnik, Golnik, Slovenia

${ }^{3}$ University Medical Centre Ljubljana, Ljubljana, Slovenia

Background: A quadricuspid aortic valve (QAV) is a rare congenital valvular malformation with common need for surgical intervention. Real time three-dimensional transthoracic echocardiography (3D TTE) may be helpful in assessment this abnormality, since complex spatial geometry, dynamic changes, and regurgitation quantification of the valve can be accurately determined ${ }^{1}$.
Case report: A 23-year-old female presented with dispnea on exertion and palpitations. Transthoracic echocardiography (TTE) revealed normal systolic function and an unusual aortic valve with fibrous thickening of cusps edges (Figure 1A) and moderate aortic regurgitation (AR) (Figure 1B). Transesophageal echocardiography (TEE) noted a QAV with two equal large cusps and two equal smaller cusps. In addition, 3D TTE was performed.

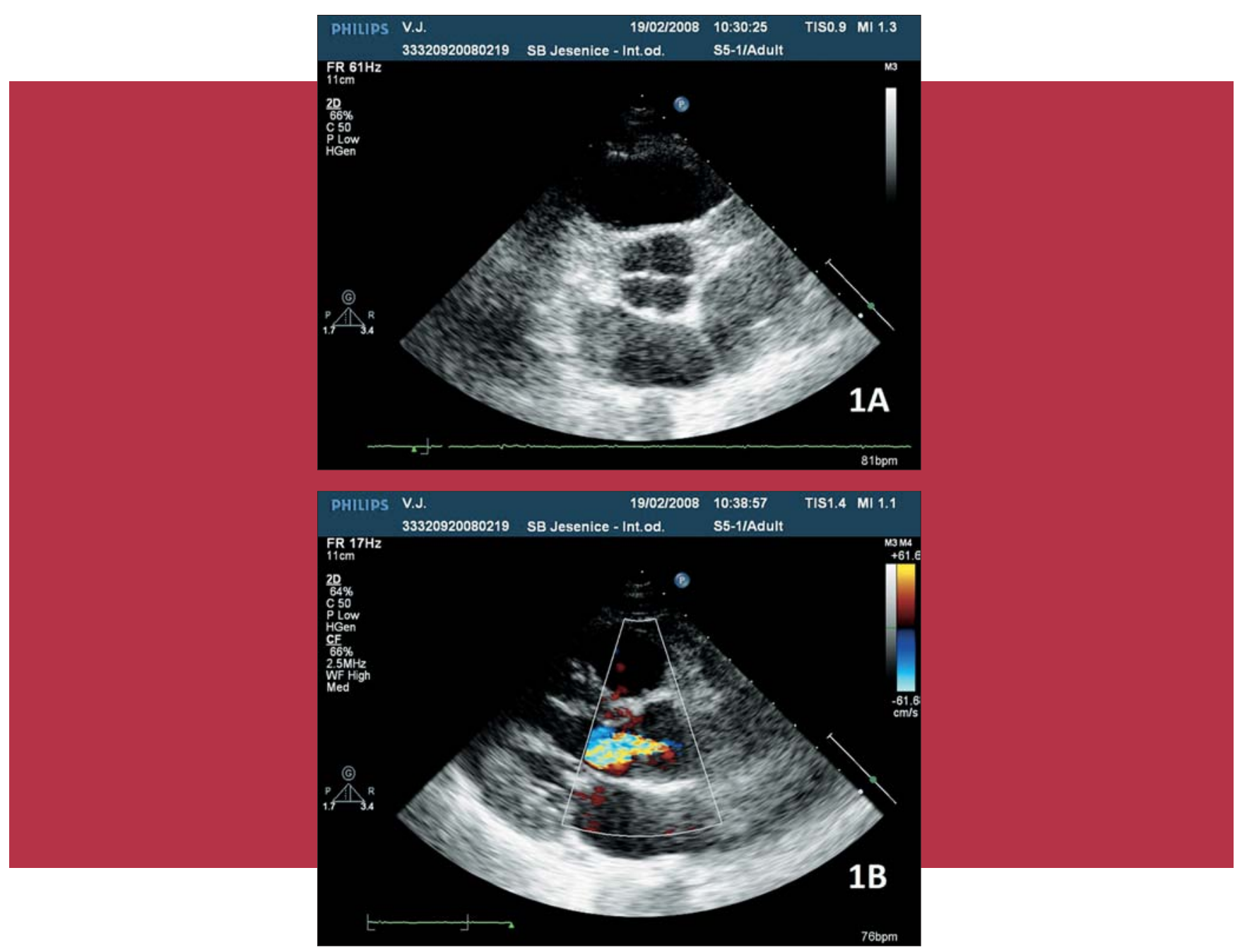

Figure 1. Fibrous thickening of cusps edges (A) and moderate aortic regurgitation (B). 
After slight tilting and adjustment of the cropping plane in the high parasternal short axis view, four cusps were clearly visualized in diastole (Figure 2A) and systole (Figure 2B). 3D color Doppler demonstrated mild aortic regurgitation
$(A R)$ with slightly eccentric jet. Low regurgitant volume of $A R$ was further confirmed with magnetic resonance imaging (MRI). Surgical intervention was not indicated.

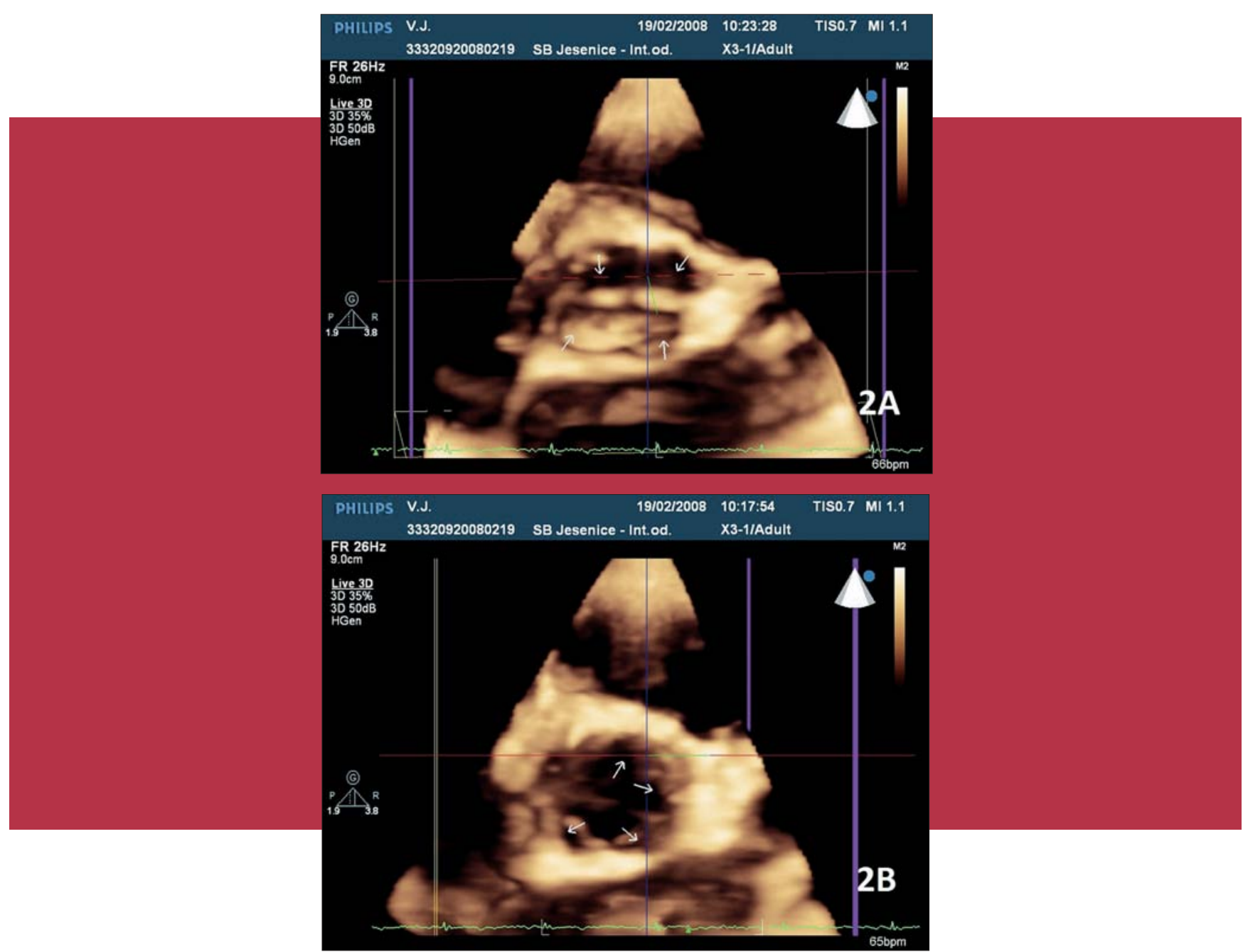

Figure 2. A four cusps obtained by three-dimensional transthoracic echocardiography in diastole (A) and in systole (B).

Discussion: Serial echocardiographic evaluations of AR seem to be an optimal diagnostic method to determine the potential need for surgical intervention. The effective regurgitant orifice area may be the most hemodynamically important parameter for quantification of AR severity, but it is difficult to derive in many cases of QAV with frequently eccentric jet. 2D TTE assessment of QAV which relies on the relationship between the size of the jet and regurgitant volume could be unreliable ${ }^{2}$. TEE is semi-invasive and not without risk. MRI derives accurate measurements, but the method is not eligible for regular follow-up. As the 3D TTE color Doppler measurements of regurgitation flow can be done in three dimensions, assessment of QAV could be more reliable in comparison to conventional echocardiography ${ }^{1,3,4}$.
Conclusion: 3D echocardiography has the potential to become the standard examination procedure for the assessment of QAV.

KEYWORDS: three-dimensional transthoracic echocardiography, quadricuspid aortic valve.

\footnotetext{
Received: $20^{\text {th }}$ Mar 2013

*Address for correspondence: Splošna bolnišnica Jesenice, Cesta Maršala Tita 112, SI-4270 Jesenice, Slovenia.

Phone: +38645868269

E-mail: janezpoklukar@gmail.com
}

\section{Literature}

1. Mumm B, Bauman R, Hyca M. Three-dimensional echo for the assessment of valvular heart disease. Cardiol Clin. 2007;25:283-95.

2. Perry GJ, Helmcke F, Nanda NC, Byard C, Soto B. Evaluation of aortic insufficiency by Doppler color flow mapping. J Am Coll Cardiol. 1987;9:952-9.

3. Fang L, Hsiung MC, Miller AP, Nanda NC, Yin WH, Young MS, et al. Assessment of aortic regurgitation by live three dimensional transthoracic echocariographic measurements of vena contracta area: usefulness and validation. Echocardiography. 2005; 22:775-81.

4. Wittlinger T, Dzemali O, Bakhtiary F, Moritz A, Kleine P. Hemodynamic evaluation of aortic regurgitation by magnetic resonance imaging. Asian Cardiovasc Thorac Ann. 2008;16(4):278-83 